\title{
A Study to Know the Knowledge, Attitude and Practices about HIV and its Related Ethical Issues among Medical Students in Karnataka
}

\author{
Kavithai $\mathrm{P}^{1}$, Anandaraj $\mathrm{R}^{2}$, Ranjitha $\mathrm{A}^{3}$, Mahabalaraju $\mathrm{Dk}^{4}$ \\ ${ }^{1}$ Dept Of Community Medicine, Government Medical College, Thiruvananthapuram, India \\ ${ }^{2}$ Dept Of Community Medicine, Indira Gandhi Medical College \& Research Center, Pondicherry, India. \\ ${ }^{3,4}$ Dept Of Community Medicine, J.J.M. Medical College, Karnataka, India
}

\begin{abstract}
:
Background: Medical students, who are future healthcare providers, should be oriented in different aspects of $H I V$, so that they inculcate healthy attitude towards PLHIV.

Objectives:

1. To study the knowledge, attitude and practices about HIV and related ethical issues among medical students.

2. To know the difference in this knowledge between first year \& third year medical students.

Methodology: A cross sectional study was conducted in September 2013 among MBBS students of first \& third years studying in J.J.M. Medical College, Davangere. Data regarding knowledge, attitude and practices and ethical issues in HIV was collected using a structured questionnaire.

Results: In a large number of medical students, knowledge, attitude, practices and ethical issues was satisfactory $(75.2 \%, 57.3 \%, 56.2 \%$ and $52.9 \%$ respectively).

Conclusion: Lacunae in the knowledge and attitude regarding ethical issues of HIV exist in considerable number of students. Though knowledge of first year students was less, ethical issues were better than third year students..
\end{abstract}

Keywords: AIDS; Ethical issues; HIV awareness; KAP; Medical students.

\section{Introduction}

Scientific \& ethical knowledge regarding HIV is an effective mode to interrupt transmission of AIDS. ${ }^{[1]}$ The ethical issues mainly revolve around the standard of care, informed consent across cultures, privacy, stigma and discrimination, protection of vulnerable groups, community consultation, ethical review mechanisms, international collaboration, epidemiological studies, clinical trials and also sociobehavioural studies on HIV/AIDS. ${ }^{[2]}$ Medical students, who are future healthcare providers, should be oriented in different aspects of HIV, so that they inculcate healthy attitude towards people living with HIV (PLHIV). Understanding the various clinical, ethical and societal concerns related to HIV/AIDS treatment and research in students, more so in fresh entrees \& students entering into final year of MBBS, is essential. This would help in development of appropriate curriculum to this target population. However, there are fewer studies that explored the knowledge and practices of medical students pertaining to HIV and related ethical issues, particularly in this region.

Hence the present study was conducted with the following objectives 1.To study the knowledge, attitude and practices about HIV and related ethical issues among medical students. 2. To know the difference in this knowledge between first year \& third year medical students.

\section{Materials and methods}

A cross sectional study was conducted in September 2013 among MBBS students of first \& third years studying in J.J.M. Medical College, Davangere, Karnataka. The purpose of the study was explained to the students before the start of the study and informed consent was obtained from them. A self administered questionnaire was developed in line with the WHO KABP questionnaire ${ }^{[3]}$ which was pretested and modified accordingly. The final questionnaire consisted of four sections, each containing 8 questions exploring the KAP and ethical issues regarding HIV. Responses to assess knowledge were recorded in the form of "true" or "false" whereas responses to other sections were recorded on a 5-point LIKERT scale, ranging from strongly disagree $(=1)$ to strongly agree $(=5)$. To avoid bias, questionnaire was simultaneously administered to the students of both the classes and those who were not present on the day of the study were excluded. Necessary steps were taken to ensure anonymity. After excluding two data forms with incomplete responses, data collected from 274 students was entered and analyzed using Microsoft EXCEL 2010. Data representation was done in percentages and proportions. Chi square test was used to find any significant difference between two qualitative variables. Level of significance was fixed at $p$ value of 0.05 . 


\section{Results}

Out of 274 students, 136 were first year MBBS and 138 were from third year. Mean age was 18.3 years and 21.3 years respectively. About 109 (39.8\%) were men and 165 (60.2\%) were women.

Among medical students; satisfactory knowledge, attitude, practice and ethical issues regarding HIV are $75.2 \%, 57.3 \%, 56.2 \%$ and $52.9 \%$ respectively. Knowledge was good in $14.2 \%$ and poor in $10.6 \%$ of the students [Fig. 1].

Knowledge of first year students was less than third year students. Ethical issues of first year students were marginally better than third year students [TABLE: 1]. There were significantly different opinions observed between first and third year MBBS students regarding HIV infectivity, mother to child transmission, stigma and condom usage [TABLE: 2].

\section{Discussion}

HIV is not only a significant public health problem but also one of the important social issues of the millennium. In our study, deficiencies in the knowledge and awareness in critical areas of the disease were noticed. Such deficiencies in knowledge of HIV do influence the future health care providers in their attitude towards their patients leading to discrimination.

A study in India identified serious gaps in the knowledge regarding HIV transmission even among medical practitioners in private and government hospitals leading to refusal of treatment to persons living with HIV. ${ }^{[4]}$ In a south Indian study conducted on first year medical students, it was noted that $25.7 \%$ believed that mosquitoes bite could transmit HIV infection ${ }^{[5]}$ while this study recorded $30.9 \%$.

Mohsin et al., in his study on MBBS students in Baroda, also showed similar misconceptions among them such as urine being potential source of HIV infection. About $40 \%$ were unwilling to assist in surgical procedures on AIDS patients. ${ }^{[6]}$ One-third of our study subjects also reported similar findings. Popular misconceptions do exist among other disciplines as well such as nursing and pharmacy students as evidenced by other authors. ${ }^{\text {[7-10] }}$

In order to impart correct knowledge about HIV transmission in medical students and to avoid discrimination of PLHIV, it is vital that HIV/AIDS related education should be imparted from the very beginning of professional education to demystify misconceptions. There should be special sessions in clinical postings. Integrated teaching in medical colleges by interdisciplinary teams and ensuring adequate clinical exposure with HIV patients would be beneficial. Health education regarding HIV in the higher secondary education level, coupled with training of school teachers will pave a long way in enforcing adequate knowledge among those entering the medical education.

Figures and Tables

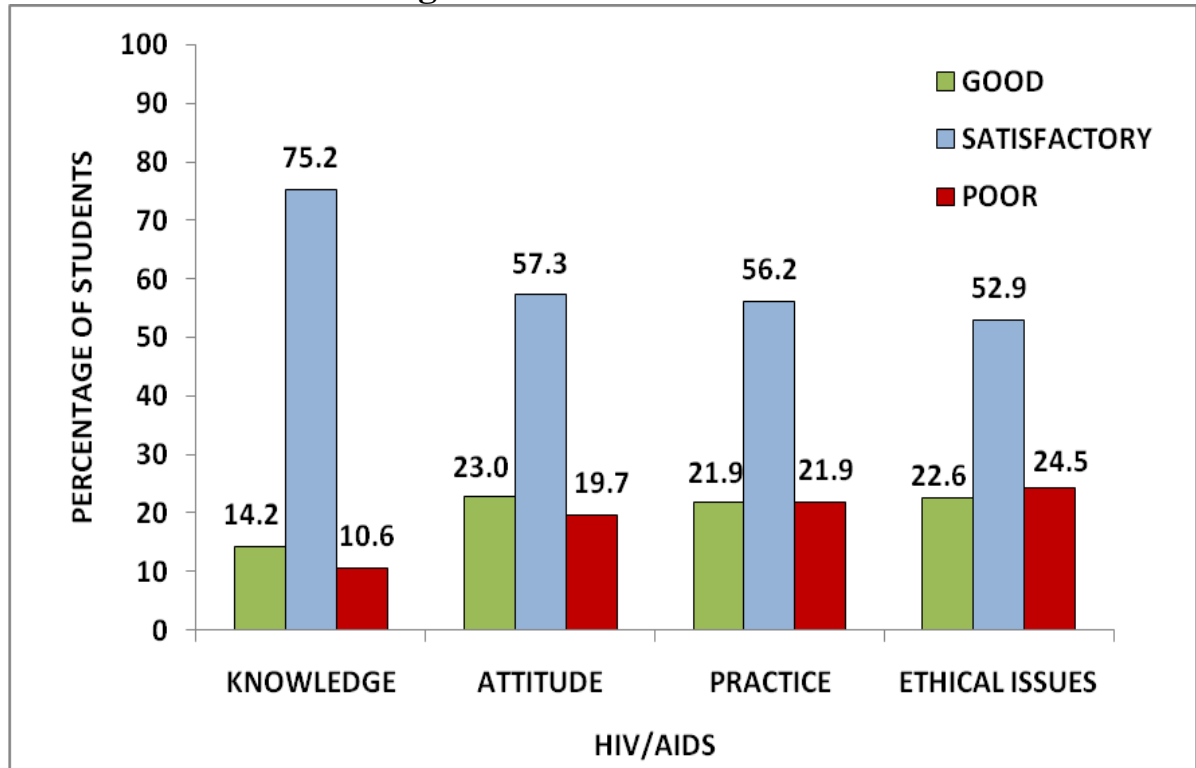

Figure 1: KAP about HIV and related ethical issues among medical students. 
Table 1: Comparison between first and third year MBBS students by scores regarding KAP about HIV and related ethical issues.

\begin{tabular}{|c|c|c|c|}
\hline \multicolumn{2}{|c|}{ Variable $^{*}$} & First Year N (\%) & Second Year N (\%) \\
\hline \multirow{3}{*}{ Knowledge } & Good & $22(16.2)$ & $37(26.8)$ \\
\cline { 2 - 4 } & Satisfactory & $106(77.9)$ & $90(65.2)$ \\
\cline { 2 - 4 } & Poor & $8(5.9)$ & $11(8.0)$ \\
\hline \multirow{3}{*}{ Attitude } & Good & $35(25.7)$ & $25(18.1)$ \\
\cline { 2 - 4 } & Satisfactory & $70(51.5)$ & $82(59.4)$ \\
\cline { 2 - 4 } & Poor & $31(22.8)$ & $31(22.5)$ \\
\hline \multirow{3}{*}{ Practice } & Good & $29(21.3)$ & $32(23.2)$ \\
\cline { 2 - 4 } & Satisfactory & $74(54.4)$ & $78(56.5)$ \\
\cline { 2 - 4 } & Poor & $33(24.3)$ & $28(20.3)$ \\
\hline \multirow{3}{*}{ Ethical Issues } & Good & $35(25.7)$ & $34(24.6)$ \\
\cline { 2 - 4 } & Satisfactory & $78(57.4)$ & $27(55.8)$ \\
\cline { 2 - 4 } & Poor & $23(16.9)$ & $27.6)$ \\
\cline { 2 - 4 } & \multicolumn{2}{|c}{}
\end{tabular}

* A scoring for each question in the KAP assessment form was done using a combined LIKERT and binary marking protocol. The scores were converted into percentiles and categorized as good (above 75th percentile), satisfactory (25th to 75 th percentile) and poor (below 25 th percentile)

Table 2: Comparison between first and third year MBBS students by certain selected facts about KAP regarding HIV and related ethical issues.

\begin{tabular}{|l|c|c|c|c|}
\hline \multicolumn{1}{|c}{ Facts } & First Year (\%) & Third Year (\%) & Chi Sq. & $P$ Value \\
\hline $\begin{array}{l}\text { Condom Is For Extramarital Affairs \& } \\
\text { Not For One-One Relationship }\end{array}$ & 29.4 & 13.7 & 6.44 & $\mathbf{0 . 0 1}$ \\
\hline $\begin{array}{l}\text { Hiv Is Transmitted By Blood Sucking } \\
\text { Insects }\end{array}$ & 30.9 & 7.9 & 15.82 & $<\mathbf{0 . 0 0}$ \\
\hline $\begin{array}{l}\text { Once Affected Its Better For A Hiv } \\
\text { Patient To Die }\end{array}$ & 9.6 & 2.9 & 2.9 & 0.08 \\
\hline $\begin{array}{l}\text { I Am Willing To Assist Or Take Care } \\
\text { Of Aids Patients In Operation } \\
\text { Theatres And Wards. }\end{array}$ & 71.3 & 68.3 & 0.1 & 0.75 \\
\hline I Feel Ashamed If He/She Had Aids & 37.5 & 17.3 & 9.42 & $\mathbf{0 . 0 0}$ \\
\hline $\begin{array}{l}\text { Hiv Patients Contracted The Disease } \\
\text { Aids By Their Immoral Activities }\end{array}$ & 55.1 & 48.2 & 0.72 & 0.39 \\
\hline $\begin{array}{l}\text { Pregnant Hiv Women Must Go For } \\
\text { Abortion }\end{array}$ & 65.4 & 37.5 & 14.63 & $\mathbf{0 . 0 0 0 1}$ \\
\hline $\begin{array}{l}\text { Hiv Patients Should Be Nursed In } \\
\text { Isolation Wards }\end{array}$ & 48.5 & 65.5 & 5.22 & $\mathbf{0 . 0 2}$ \\
\hline $\begin{array}{l}\text { Hiv Infected Surgeon Should Not Be } \\
\text { Allowed To Do A Surgery }\end{array}$ & 62.5 & 69.8 & 0.9 & 0.34 \\
\hline Hbv Is More Infective Than Hiv & 35.3 & 73.4 & 28.01 & $<\mathbf{0 . 0 0}$ \\
\hline
\end{tabular}

\section{Conclusion}

Lacunae in the knowledge and attitude regarding ethical issues of HIV exist in a considerable number of medical students.

\section{Acknowledgements}

The authors thank the postgraduates and interns of the department for their valuable assistance in data collection. We also thank our students without whom this work would have not been possible.

\section{References}

[1]. Park K. Park's Text book of Preventive and Social Medicine.23rd ed. Jabalpur: Banarsidas Bhanot; 2015.

[2]. Muthuswamy V. Ethical issues in HIV/AIDS research. Indian J Med Res. 2005 Apr; 121(4):601-10.

[3]. WHO. Research Package: Knowledge, Attitude, Beliefs and practices on AIDS (KABP) phase I. WHO, Geneva, 1990.

[4]. Daniel M. Aids in India: Denial and disaster. Harvard International Review. 2003; Vol. 25, 9-10.

[5]. Kuruvila M, Venugopalan PP, Sridhar KS, Kumar P, Rao GS, Kotian S. KAP study on HIV / AIDS among first year MBBS students. Indian J Dermatol Venereol Leprol. 1997;63:225-8.

[6]. Mohsin S, Nayak S, Mandaviya V. Medical students' knowledge and attitudes related to HIV/AIDS. National Journal of Community medicine.2010;1(2):146-149.

[7]. Dobe, M. (1995). Awareness on AIDS among health care professionals. Indian Public Health, 39, 3, 105-108

[8]. Kumar, A., Lal, P., Ingle, G.K., \& Gulati, N. (1999). AIDS-related apprehensions among nursing students of Delhi. J Comm. Dis., $31,4,217-21$.

[9]. Singh SK, Saxena A, Krishna G. A profile of HIV infection/AIDS related knowledge among female students of Kanpur district, India. Kathmandu Univ Med J. 2007;5:27-31.

[10]. Mizanur M, Kabir M, Shahidullah M. Adolescent knowledge and awareness about AIDS/HIV and factors affecting them in Bangladesh. J Ayub Med Coll Abbottabad. 2009; 21:3-6. 